\title{
Monotone Boolean Functions with s Zeros Farthest from Threshold Functions
}

\author{
Kazuyuki Amano ${ }^{1}$ and Jun Tarui ${ }^{2}$ \\ ${ }^{1}$ Graduate School of Information Sciences, Tohoku University, Sendai 980-8579 Japan \\ amadecei.tohoku.ac.jp \\ ${ }^{2}$ Department of Information and Communication Engineering, University of Electro-Communications \\ Chofu, Tokyo 182-8585 Japan tarui@ice.uec.ac.jp
}

Let $T_{t}$ denote the $t$-threshold function on the $n$-cube: $T_{t}(x)=1$ if $\left|\left\{i: x_{i}=1\right\}\right| \geq t$, and 0 otherwise. Define the distance between Boolean functions $g$ and $h, d(g, h)$, to be the number of points on which $g$ and $h$ disagree. We consider the following extremal problem: Over a monotone Boolean function $g$ on the $n$-cube with $s$ zeros, what is the maximum of $d\left(g, T_{t}\right)$ ? We show that the following monotone function $p_{s}$ maximizes the distance: For $x \in\{0,1\}^{n}$, $p_{s}(x)=0$ if and only if $N(x)<s$, where $N(x)$ is the integer whose $n$-bit binary representation is $x$. Our result generalizes the previous work for the case $t=\lceil n / 2\rceil$ and $s=2^{n-1}$ by Blum, Burch, and Langford [BBL98-FOCS98], who considered the problem to analyze the behavior of a learning algorithm for monotone Boolean functions, and the previous work for the same $t$ and $s$ by Amano and Maruoka [AM02-ALT02].

\section{Introduction and Overview}

For a Boolean function $h$ and a class $\mathcal{C}$ of Boolean functions, we consider the following extremal problem: what is the maximum distance between $g \in \mathcal{C}$ and $h$ ? Equivalently, under the uniform distribution on $\{0,1\}^{n}$, how small can the correlation between $g \in \mathcal{C}$ and $h$ be? The distance between Boolean functions $g$ and $h, d(g, h)$, is defined to be the number of points on which $g$ and $h$ disagree. A Boolean function $g:\{0,1\}^{n} \rightarrow\{0,1\}$ is monotone if, for $x, y \in\{0,1\}^{n}, x \leq y \Rightarrow g(x) \leq g(y)$, where for $x, y \in\{0,1\}^{n}$, $x \leq y$ if and only if $x_{i} \leq y_{i}$ for all $i=1, \ldots, n$. A Boolean function is fair if it outputs 1 on exactly half of its inputs. The starting point of our work is the fact that among all fair monotone Boolean functions, $a$ single variable function $g(x)=x_{i}$ is farthest from the majority function; this was conjectured by Blum, Burch, and Langford [BBL98], and was proved by Amano and Maruoka [AM02].

The main concern of the work of Blum, Burch, and Langford is learning of monotone Boolean functions. They gave the following simple algorithm for weakly learning a monotone Boolean function under the uniform distribution: Given samples $\left(x_{1}, g\left(x_{1}\right)\right),\left(x_{2}, g\left(x_{2}\right)\right), \ldots,\left(x_{m}, g\left(x_{m}\right)\right)$, output, as a hypothesis, a function that is most correlated with those samples among three functions $\{0,1$, Majority $\}$, where 0 and 1 are constant functions. With high probability the output of the algorithm has correlation at least $\Omega(1 / \sqrt{n})$ with $g$. Blum, Burch, and Langford showed, using the Kruskal-Katona theorem about the minimum size of a shadow, that any fair monotone Boolean function $g$ has correlation at least $\Omega(1 / \sqrt{n})$ with Majority. They conjectured that in fact a single variable function $g(x)=x_{i}$ is a fair monotone function 1365-8050 (c) 2005 Discrete Mathematics and Theoretical Computer Science (DMTCS), Nancy, France 
that is farthest from Majority. Amano and Maruoka proved this conjecture also using the Kruskal-Katona theorem.

In this paper we give a generalization in which we consider any threshold function, not just Majority, and any monotone function with a prescribed number of zeros, not just a fair one. Our proof is selfcontained; we do not use the Kruskal-Katona theorem.

Let $T_{t}$ denote the $t$-threshold function: for $x \in\{0,1\}^{n}, T_{t}(x)=1$ if $\left|\left\{i: x_{i}=1\right\}\right| \geq t$, and 0 otherwise. Throughout the paper $t$ is an integer; it will be convenient to allow $t$ to be negative; for $t \leq 0$, $T_{t}$ is the constant 1 function on the $n$-cube. The majority function is function $T_{t}$ with $t=\lceil n / 2\rceil$. For a Boolean function $g$, let $\sharp_{0}(g)$ and $\sharp_{1}(g)$ respectively denote the number of points on which $g=0$ and on which $g=1$. Similarly, for Boolean functions $g$ and $h$, and $a, b \in\{0,1\}$, let $\sharp_{a b}(g, h)$ denote the number of points $x$ such that $g(x)=a$ and $h(x)=b$.

The problem we consider is the following: Among all monotone $g:\{0,1\}^{n} \rightarrow\{0,1\}$ with $\sharp_{0}(g)=s$ $\left(0 \leq s<2^{n}\right)$, what is the maximum of $d\left(g, T_{t}\right)$ ? Maximizing $d(g, h)$ for a fixed function $h$ and a function $g$ with $\sharp_{0}(g)=s$ is equivalent to maximizing $\sharp_{01}(g, h)$ since for any $g$ and $h, d(g, h)=\sharp_{0}(h)-$ $\sharp_{0}(g)+2 \sharp_{01}(g, h)$. For $0 \leq s<2^{n}$, define the monotone function $p_{s}:\{0,1\}^{n} \rightarrow\{0,1\}$ as follows. For $x \in\{0,1\}^{n}, p_{s}(x)=0$ if and only if $N(x)<s$, where $N(x)$ is the integer whose $n$-bit binary representation is $x$.

Theorem Let $g:\{0,1\}^{n} \rightarrow\{0,1\}$ be monotone with $\sharp_{0}(g)=s$. Then, for any integer $t$,

$$
\sharp_{01}\left(g, T_{t}\right) \leq \sharp_{01}\left(p_{s}, T_{t}\right), \quad \text { and hence } d\left(g, T_{t}\right) \leq d\left(p_{s}, T_{t}\right) .
$$

\section{Proof of Theorem}

For an integer $i \geq 0$, let $b(i)$ be the number of 1's in the binary representation of the integer $i$. Let $0 \leq l \leq m$. For an integer $t$, define $f_{t}(l, m)$ to be the number of integers $i$ such that $l \leq i<m$ and $b(i) \geq t$. Note that for $t \leq 0, f_{t}(l, m)=f_{0}(l, m)=m-l$. Also note that the following hold: $f_{t}(l, m) \leq f_{t-1}(l, m)$; for $0 \leq k<2^{r}, f_{t}(0, k)=f_{t-1}\left(2^{r}, 2^{r}+k\right) ; \sharp_{01}\left(p_{s}, T_{t}\right)=f_{t}(0, s)$.

It turns out that the main work we do to prove the theorem is an analysis of $f_{t}(l, m)$. This aspect of our proof is somewhat similar to a proof of the edge-isoperimetric inequality on the Boolean cube explained in the book by Bollobas [Bo86, $\S 16$ Isoperimetric Problems]. We now state a key lemma, Lemma 1, and two auxiliary lemmas, Lemmas 2 and 3. We wll give a proof of the theorem using Lemma 1, and then give proofs of the three lemmas.

Lemma 1 For $0 \leq l \leq m$ and any integer $t$,

$$
f_{t}(0, m)+f_{t-1}(0, l) \leq f_{t}(0, m+l) .
$$

Lemma 2 For $k, l \geq 0$ and any integer $t$,

$$
f_{t}(0, k) \leq f_{t}(l, l+k) .
$$

Lemma 3 For $k, l, q \geq 0$ such that $l+k \leq 2^{q}$ and for any integer $t$,

$$
f_{t}(l, l+k) \leq f_{t}\left(2^{q}-k, 2^{q}\right) .
$$


Proof of Theorem using Lemma 1. The proof is by induction on $n$. The base case $n=1$ is trivial. For induction assume that $n>1$ and that the assertion holds for $n-1$. Let $g_{0}$ and $g_{1}$ be the Boolean functions on the $(n-1)$-cube obtained from $g$ by fixing the first bit to be 0 and 1 respectively; i.e., for $e=0,1$, and for $x \in\{0,1\}^{n-1}, g_{e}(x)=g(e x)$. Let $m=\sharp_{0}\left(g_{0}\right)$ and $l=\sharp_{0}\left(g_{1}\right)$. Clearly, $m+l=s$, and since $g$ is monotone, $m \geq l$. Thus we have

$$
\begin{aligned}
\sharp_{01}\left(g, T_{t}\right) & =\sharp_{01}\left(g_{0}, T_{t}\right)+\sharp_{01}\left(g_{1}, T_{t-1}\right) \\
& \leq \sharp_{01}\left(p_{m}, T_{t}\right)+\sharp_{01}\left(p_{l}, T_{t-1}\right) \\
& =f_{t}(0, m)+f_{t-1}(0, l) \\
& \leq f_{t}(0, m+l) \\
& =\sharp_{01}\left(p_{s}, T_{t}\right),
\end{aligned}
$$

where the first inequality is by the inductive assumption and the second inequality is by Lemma 1 .

Proofs of Lemmas 2 and 3. For $0 \leq i<2^{q}$, the $q$-bit binary representation of $i$ has bit 1 at position $j$ $(1 \leq j \leq q)$ if and only if the $q$-bit binary representation of $2^{q}-1-i$ has bit 0 at position $j$. Hence Lemma 2 readily yields Lemma 3 .

Now we prove Lemma 2. The assertion is trivial when $l=k=0$. Assume that $l+k \geq 1$ and let $r=\left\lfloor\log _{2}(l+k)\right\rfloor$ so that we have $2^{r} \leq l+k<2^{r+1}$. The proof is by induction on $r$; more precisely, we prove Lemma 2 by inductively assuming that the assertion of Lemma 2 holds and the corresponding assertion of Lemma 3 holds.

In the base case when $r=0$, we have $l+k=1$ and thus either (i) $l=0, k=1$ or (ii) $l=1, k=0$; in both cases the claim is immediate. For induction assume that $r>0$ and that for $r-1$ the assertion holds, and hence the corresponding assertion of Lemma 3 also holds.

CASE 1: $2^{r} \leq l: \quad$ In this case $2^{r} \leq l \leq l+k<2^{r+1}$ and

$$
f_{t}(l, l+k)=f_{t-1}\left(l-2^{r}, l+k-2^{r}\right) \geq f_{t-1}(0, k) \geq f_{t}(0, k),
$$

where the first inequality is by the inductive assumption.

CASE 2: $l<2^{r}$ and $k<2^{r}$ :

$$
\begin{aligned}
f_{t}(l, l+k) & =f_{t}\left(l, 2^{r}\right)+f_{t}\left(2^{r}, l+k\right) \\
& =f_{t}\left(l, 2^{r}\right)+f_{t-1}\left(0, l+k-2^{r}\right) \\
& \geq f_{t}\left(l+k-2^{r}, k\right)+f_{t-1}\left(0, l+k-2^{r}\right) \\
& \geq f_{t}\left(l+k-2^{r}, k\right)+f_{t}\left(0, l+k-2^{r}\right) \\
& =f_{t}(0, k),
\end{aligned}
$$

where the first inequality is by Lemma 3 . 
CASE 3: $l<2^{r}$ and $k \geq 2^{r}:$ In this case $l<2^{r} \leq 2^{r}+l \leq l+k$ and

$$
\begin{aligned}
f_{t}(l, l+k) & =\left[f_{t}\left(l, 2^{r}\right)+f_{t}\left(2^{r}, 2^{r}+l\right)\right]+f_{t}\left(2^{r}+l, l+k\right) \\
& =\left[f_{t}\left(l, 2^{r}\right)+f_{t-1}(0, l)\right]+f_{t}\left(2^{r}+l, l+k\right) \\
& \geq\left[f_{t}(0, l)+f_{t}\left(l, 2^{r}\right)\right]+f_{t}\left(2^{r}+l, l+k\right) \\
& =f_{t}\left(0,2^{r}\right)+f_{t}\left(2^{r}+l, l+k\right) \\
& =f_{t}\left(0,2^{r}\right)+f_{t-1}\left(l, l+k-2^{r}\right) \\
& \geq f_{t}\left(0,2^{r}\right)+f_{t-1}\left(0, k-2^{r}\right) \\
& =f_{t}\left(0,2^{r}\right)+f_{t}\left(2^{r}, k\right) \\
& =f_{t}(0, k),
\end{aligned}
$$

where the second inequality is by the inductive assumption.

Proof of Lemma 1. The asertion is trivial when $l=m=0$. Asume that $m \geq 1$ and let $r=\left\lfloor\log _{2} m\right\rfloor$ so that we have $2^{r} \leq m<2^{r+1}$. The proof is by induction on $r$. In the base case when $r=0$ we have $m=1$, and $l=0$ or $l=1$; in both cases the claim is immediate. For induction assume that $r>0$ and that the claim holds for $r-1$.

CASE 1: $2^{r} \leq l$ :

$$
\begin{aligned}
f_{t}(0, m)+f_{t-1}(0, l) & =f_{t}\left(0,2^{r}\right)+f_{t}\left(2^{r}, m\right)+f_{t-1}\left(0,2^{r}\right)+f_{t-1}\left(2^{r}, l\right) \\
& =f_{t}\left(0,2^{r}\right)+f_{t-1}\left(0, m-2^{r}\right)+f_{t-1}\left(0,2^{r}\right)+f_{t-2}\left(0, l-2^{r}\right) \\
& =f_{t}\left(0,2^{r}\right)+f_{t-1}\left(0,2^{r}\right)+f_{t-1}\left(0, m-2^{r}\right)+f_{t-2}\left(0, l-2^{r}\right) \\
& \leq f_{t}\left(0,2^{r}\right)+f_{t-1}\left(0,2^{r}\right)+f_{t-1}\left(0, m+l-2^{r+1}\right) \\
& =f_{t}(0, m+l),
\end{aligned}
$$

where the inequality is by the inductive assumption.

CASE 2: $l<2^{r},\left(m-2^{r}\right)+l \leq 2^{r}$ :

$$
\begin{aligned}
f_{t}(0, m)+f_{t-1}(0, l) & =f_{t}\left(0,2^{r}\right)+f_{t}\left(2^{r}, m\right)+f_{t-1}(0, l) \\
& =f_{t}\left(0,2^{r}\right)+f_{t-1}\left(0, m-2^{r}\right)+f_{t-1}(0, l) \\
& \leq f_{t}\left(0,2^{r}\right)+f_{t-1}\left(0, m-2^{r}\right)+f_{t-1}\left(m-2^{r}, m-2^{r}+l\right) \\
& =f_{t}\left(0,2^{r}\right)+f_{t-1}\left(0, m-2^{r}+l\right) \\
& =f_{t}\left(0,2^{r}\right)+f_{t}\left(2^{r}, m+l\right) \\
& =f_{t}(0, m+l),
\end{aligned}
$$

where the inequality is by Lemma 2 . 
CASE 3: $l<2^{r}, m+l>2^{r+1}$ : We have the following derivation where Lemma 3 is used in the form (1) for the inequality below.

$$
f_{t-1}\left((m+l)-2^{r+1}, l\right) \leq f_{t-1}\left(m-2^{r}, 2^{r}\right)
$$

$$
\begin{aligned}
& f_{t}(0, m)+f_{t-1}(0, l) \\
= & f_{t}\left(0,2^{r}\right)+f_{t}\left(2^{r}, m\right)+f_{t-1}\left(0,(m+l)-2^{r+1}\right)+f_{t-1}\left((m+l)-2^{r+1}, l\right) \\
= & f_{t}\left(0,2^{r}\right)+f_{t-1}\left(0, m-2^{r}\right)+f_{t-1}\left(0,(m+l)-2^{r+1}\right)+f_{t-1}\left((m+l)-2^{r+1}, l\right) \\
\leq & f_{t}\left(0,2^{r}\right)+f_{t-1}\left(0, m-2^{r}\right)+f_{t-1}\left(m-2^{r}, 2^{r}\right)+f_{t-1}\left(0,(m+l)-2^{r+1}\right) \\
= & f_{t}\left(0,2^{r}\right)+f_{t-1}\left(0,2^{r}\right)+f_{t-1}\left(0,(m+l)-2^{r+1}\right) \\
= & f_{t}\left(0,2^{r}\right)+f_{t}\left(2^{r}, 2^{r+1}\right)+f_{t}\left(2^{r+1}, m+l\right) \\
= & f_{t}(0, m+l) .
\end{aligned}
$$

\section{Acknowledgements}

The authors thank the anonymous referees for helpful comments.

\section{References}

[AM02] K. Amano and A. Maruoka. On Learning Monotone Boolean Functions under the Uniform Distribution, Proc. of ALT02: The 13th International Conference on Algorithmic Learning Theory: Lecture Notes in Computer Science, vol. 2533, pp. 57-68, Springer-Verlag, 2002; a journal version to appear in Theoretical Computer Science.

[BBL98] A. Blum, C. Burch, and L. Langford. On Learning Monotone Boolean Functions, Proc. of FOCS98: The 39th Annual IEEE Symposium on Foundations of Computer Science, pp. 408415, IEEE Press, 1998.

[Bo86] B. Bollobas. Combinatorics, Cambridge University Press, 1986. 
